\title{
Pancreas-oriented superior mesenteric vein dissection for D3 lymphadenectomy in laparoscopic right hemicolectomy
}

\author{
W.-J. Meng ${ }^{1} \cdot$ X.-B. Deng ${ }^{1} \cdot$ Y.-C. Zhang ${ }^{2} \cdot$ M.-T. Wei ${ }^{1} \cdot$ C.-W. Jin ${ }^{3} \cdot$ \\ Z.-Q. Wang ${ }^{1}$
}

Received: 12 June 2016/ Accepted: 13 November 2016/Published online: 5 December 2016

(C) Springer International Publishing AG 2016

Laparoscopic right hemicolectomy has been demonstrated to improve short-term postoperative results without compromising long-term oncological results. However, it remains the most challenging and time-consuming procedure because of the technical difficulties in D3 lymphadenectomy along the superior mesenteric vein (SMV), with a reported mean operating time of 150-288 min. Here, we present a video demonstration of a novel procedure for D3 lymphadenectomy, characterized by unidirectionally progressive dissection along the SMV and with the pancreas as the destination for each round. First, the SMV is identified where it is joined by the ileocolic vein or at a level distal to that, and the peritoneum is cut along left side of the SMV until the pancreatic neck is displayed and the lesser omentum is entered. Then, ileocolic, right and middle colic veins (MCVs) are dissected with skeletonizing of the ventral aspect of the SMV caudally to cranially. After the MCV is dissected and the whole of pancreatic neck exposed, the ileocolic and right colic vein are ligated successively at their origins. Finally, an anastomosis is

W.-J. Meng and X.-B. Deng have contributed equally to this work.

Electronic supplementary material The online version of this article (doi:10.1007/s10151-016-1556-x) contains supplementary material, which is available to authorized users.

Z.-Q. Wang

wangzqzyh@163.com

1 Department of Gastrointestinal Surgery, West China Hospital of Sichuan University, No. 37, Guoxue Street,

Chengdu 610041, China

2 Department of Gastrointestinal Surgery, The 3rd Hospital of Chengdu, Chengdu, China

3 Department of General Surgery, The 5th Hospital of Chengdu, Chengdu, China created extracorporeally. This procedure is based on the anatomical characteristics of the venous system: No tributaries join the left one-third of SMV anteriorly. It facilitates the exposure of the whole course of the SMV on its left side, which may effectively avoid injury to the frequently variable MCV or Henles' trunk because they are not treated until the whole of pancreatic neck is displayed.

In the period from February 2013 and December 2014, we treated 21 patients with right-sided colon cancer using this unidirectional procedure. Institutional ethics committee approval was obtained, and all patients gave written informed consent. The operation time was $166 \pm 27.1 \mathrm{~min}$ (120-200 min), and blood loss was $45 \pm 27.7 \mathrm{ml}$ (20-100 ml). The number of harvested lymph nodes was $26 \pm 14.1$ (range 5-58 nodes), with a total of 15 (range 1-3) metastatic lymph nodes in 10 patients. This procedure appears to be a feasible and safe alternative for D3 lymphadenectomy in laparoscopic right hemicolectomy.

Compliance with ethical standards

Conflict of interest The authors declare that they have no conflict of interest.

Ethical approval This study complied with all ethical standards.

Informed consent Informed consent was obtained from the patient. 